PROCEEDINGS OF THE

AMERICAN MATHEMATICAL SOCIETY

Volume 126, Number 2, February 1998, Pages 499-506

S $0002-9939(98) 04070-2$

\title{
AVERAGES OF OPERATORS AND THEIR POSITIVITY
}

\author{
MASARU NAGISA AND SHUHEI WADA \\ (Communicated by Palle E. T. Jorgensen)
}

\begin{abstract}
Let $T$ be a bounded linear operator on a Hilbert space. We prove that $T$ is positive, if there exists a positive integer $N$ such that

$$
\left\|I-\frac{1}{N+1} \sum_{i=k}^{k+N} T^{i}\right\|,\left\|I-\frac{1}{N+2} \sum_{i=k}^{k+N+1} T^{i}\right\| \leq 1
$$

for any non-negative integer $k$. For several commuting operators, we can extend this result and get the similar statement.
\end{abstract}

\section{INTRODUCTION}

Let $T$ be a bounded linear operator on a Hilbert space $\mathcal{H}$. If $\left\|I-T^{n}\right\| \leq \delta<1$ for all $n \in \mathbb{N}$, then $T=I$. This fact is well-known as the theorem of Cox $([1],[6])$. For several power bounded operators $T_{1}, T_{2}, \ldots, T_{n}$, the authors show the following statement as an extension of the above one:

$$
\left\|I-\frac{1}{|d(n, k)|} \sum_{\vec{d} \in d(n, k)} T_{1}^{d_{1}} T_{2}^{d_{2}} \cdots T_{n}^{d_{n}}\right\| \leq \delta<1 \text { implies } T_{1}=T_{2}=\cdots=T_{n}=I,
$$

where $d(n, k)=\left\{\vec{d}=\left(d_{1}, d_{2}, \ldots, d_{n}\right) \mid d_{i} \geq 0, \sum_{i=1}^{n} d_{i}=k\right\} \quad(k \geq 0)$ and $|d(n, k)|$ is the cardinal number of the set $d(n, k)$.

In the above statement, the distance between averages and the identity is less than 1. In this paper we treat the case that the distance is less than or equal to 1 , and we get the following result:

$$
\begin{aligned}
& (*) 0 \leq T \leq I \text { if }\left\|I-T^{n}\right\| \leq 1 \text { for all } n \in \mathbb{N}, \\
& (* *) 0 \leq T_{i}^{N} \leq I \text { if }\left\|I-\frac{1}{|d(n, k)|} \sum_{\vec{d} \in d(n, k)} T_{1}^{d_{1}+e_{1}} T_{2}^{d_{2}+e_{2}} \cdots T_{n}^{d_{n}+e_{n}}\right\| \leq 1
\end{aligned}
$$

for all $k \geq N, e_{1}, e_{2}, \ldots, e_{n} \in \mathbb{N} \cup\{0\}$, where $T_{1}, T_{2}, \ldots, T_{n}$ are commuting operators (i.e., $T_{i} T_{j}=T_{j} T_{i}$ ).

Received by the editors April 30, 1996 and, in revised form, August 12, 1996.

1991 Mathematics Subject Classification. Primary 47B65; Secondary $47 B 44$.

(C)1998 American Mathematical Society 


\section{Positive contractions}

For a bounded linear operator $T$ on $\mathcal{H}$, we define the numerical range $W(T)$ of $T$ by

$$
W(T)=\{(T x \mid x) \mid x \in \mathcal{H},\|x\|=1\} .
$$

T. Kato [4] shows that $W(f(T))$ is contained in the closed convex hull of $f(\Pi)$ whenever $f$ is holomorphic on a neighborhood of the closed right half plane $\Pi$ of $\mathbb{C}$ and $W(T)$ is contained in $\Pi$. Using this fact, C. R. de Prima and B. K. Richard [2] show that $T$ is positive whenever $W\left(T^{n}\right)$ is contained in $\Pi$ for all $n \in \mathbb{N}$. Concerning the statement $(*)$ we prove the following theorem.

Theorem 1. Let $T$ be an operator on a Hilbert space $\mathcal{H}$. Then the following are equivalent:

(1) $0 \leq T \leq I$.

(2) $\left\|I-T^{n}\right\| \leq 1$ for all $n \in \mathbb{N}$.

(3) There exists a positive integer $N$ such that

$$
\left\|I-\frac{1}{n+1} \sum_{j=k}^{k+n} T^{j}\right\| \leq 1
$$

for all $n \geq N$ and $k \in \mathbb{N} \cup\{0\}$.

(4) There exists a positive integer $N$ such that

$$
\left\|I-\frac{1}{n+1} \sum_{j=k}^{k+n} T^{j}\right\| \leq 1
$$

for $n \in\{N, N+1\}$ and $k \in \mathbb{N} \cup\{0\}$.

Proof. The implications $(1) \Rightarrow(2) \Rightarrow(3) \Rightarrow(4)$ are immediate.

(2) $\Rightarrow(1)$ For $x \in \mathcal{H}$ with $\|x\|=1$, we have

$$
\left|1-\left(T^{n} x \mid x\right)\right|=\left|\left(\left(I-T^{n}\right) x \mid x\right)\right| \leq\left\|I-T^{n}\right\| \leq 1,
$$

so $W\left(T^{n}\right)$ is contained in $\Pi$ for all $n \in \mathbb{N}$. This means that $T$ is positive. If there exists a positive number $t \in S p(T)$ such that $t>1$, then there exists a positive integer $n$ such that $t^{n}>2$. So we have that the spectral radius of $I-T^{n}$ is more than 1 . This contradicts $\left\|I-T^{n}\right\| \leq 1$. Thus we have $0 \leq T \leq I$.

$(4) \Rightarrow(1)$ We define $T(n, k)$ by

$$
T^{k}\left(\frac{1}{n+1} \sum_{j=0}^{n} T^{j}\right) \text { for any } n \in \mathbb{N} \text { and } k \in \mathbb{N} \cup\{0\} .
$$

For any $m \geq 2, T(n, k)^{m}$ is represented as a convex combination of elements in $\{T(n, l) \mid l \in \mathbb{N} \cup\{0\}\}$. In fact,

$$
T(n, k)^{m}=\frac{1}{(n+1)^{m-1}} \sum_{j_{1}, j_{2}, \ldots, j_{m-1}=0}^{n} T\left(n, m k+j_{1}+\cdots+j_{m-1}\right) .
$$

So we have $\left\|I-T(N, k)^{m}\right\| \leq 1$ and $\left\|I-T(N+1, k)^{m}\right\| \leq 1$. By the implication $(2) \Rightarrow(1), T(N, k)$ and $T(N+1, k)$ are positive. Since $T=(N+2) T(N+1,1)-$ 
$(N+1) T(N, 2), T$ is self-adjoint. For a real number $r$, the number $r$ belongs to the closed interval [0,1], if $r$ satisfies

$$
\left|1-r^{k} \frac{1}{n+1} \sum_{j=0}^{n} r^{j}\right| \leq 1 \quad \text { for all } n=N, N+1 \text { and } k \geq 0 .
$$

Therefore $0 \leq T \leq I$.

Remark. The implication $(2) \Rightarrow(1)$ is suggested by Y. Nakamura. In relation to the statement of the above Theorem, we also note the following fact. Let $\omega=\frac{-1+\sqrt{3} i}{2}$, and set $T=\omega I$. By simple calculation, we have

$$
\begin{gathered}
\left\|I-\frac{1}{3} \sum_{j=k}^{k+2} T^{j}\right\|=1 \quad \text { for all } k \in \mathbb{N} \cup\{0\} \\
\left\|I-\frac{1}{n+1} \sum_{j=0}^{n} T^{j}\right\| \leq 1 \quad \text { for all } n \in \mathbb{N} .
\end{gathered}
$$

Then $T$ is not positive, nor self-adjoint.

\section{Nilpotent operators AND positive CONTRACTIONS}

Proposition 2. Let $T$ be a bounded linear operator on a Hilbert space $\mathcal{H}$ and $n$ a positive integer. Then the following are equivalent:

(1) $T^{n}$ and $T^{n+1}$ are positive contractions.

(2) $T$ is a direct sum of operators $N$ and $S$ such that

$$
N^{n}=0, \quad 0 \leq S \leq I .
$$

(3) For any integer $m \geq n,\left\|I-T^{m}\right\| \leq 1$.

Proof. The implication $(2) \Rightarrow(3)$ is trivial, and the implication $(3) \Rightarrow(1)$ follows from Theorem 1. So it suffices to show $(1) \Rightarrow(2)$. At first we remark that $\operatorname{KerT} T^{n}=$ $\operatorname{Ker} T^{n+1}$, because

$$
\operatorname{Ker} T^{n} \subset K \operatorname{Ker} T^{n+1} \subset \cdots \subset K \operatorname{Ker} T^{2 n}=\operatorname{Ker}\left(T^{n}\right)^{*} T^{n}=\operatorname{Ker} T^{n} .
$$

We decompose the Hilbert space $\mathcal{H}$ into the direct sum of $\operatorname{Ker} T^{n}$ and $\left(\operatorname{Ker} T^{n}\right)^{\perp}=$ $\overline{\operatorname{Range}\left(T^{n}\right)}$. Then $T, T^{n}$ and $T^{n+1}$ have the form

$$
T=\left(\begin{array}{ll}
A & B \\
C & D
\end{array}\right), \quad T^{n}=\left(\begin{array}{cc}
0 & 0 \\
0 & E
\end{array}\right), \quad T^{n+1}=\left(\begin{array}{cc}
0 & 0 \\
0 & F
\end{array}\right)
$$

with respect to the decomposition $\left(K \operatorname{er} T^{n}\right) \oplus\left(\operatorname{Ker} T^{n}\right)^{\perp}$. By the calculation

$$
\begin{aligned}
T^{n+1} & =T T^{n}=\left(\begin{array}{cc}
0 & B E \\
0 & D E
\end{array}\right) \\
& =T^{n} T=\left(\begin{array}{cc}
0 & 0 \\
E C & E D
\end{array}\right),
\end{aligned}
$$

we have $B E=0, E C=0$ and $D E=E D=F=F^{*}=(E D)^{*}=D^{*} E$. Since $\overline{\text { Range }(E)}=(\operatorname{Ker} E)^{\perp}=\left(\operatorname{Ker}^{n}\right)^{\perp}, B=0, C=0$ and $D=D^{*}$. In particular, we have $A^{n}=0$. By the positivity and contactivity of $T^{n}$ and $T^{n+1}$, we can get that $D$ is positive and contractive. 
The following statement is a direct result of the above proof.

Corollary 3. Let $T$ be a bounded linear operator on a Hilbert space $\mathcal{H}$ and $n$ a positive integer. Then the following are equivalent:

(1) $T^{n}$ and $T^{n+1}$ are self-adjoint.

(2) $T$ is a direct sum of operators $N$ and $S$ such that

$$
N^{n}=0, \quad S=S^{*} .
$$

Let $T_{1}, T_{2}, \ldots, T_{n}$ be bounded linear operators on a Hilbert space $\mathcal{H}$. In the rest of the paper, we assume that operators $T_{1}, T_{2}, \ldots, T_{n}$ are commuting with each other. We define the set $d(n, k)$ by

$$
d(n, k)=\left\{\vec{d}=\left(d_{1}, d_{2}, \cdots, d_{n}\right) \mid d_{i} \in \mathbb{N} \cup\{0\}, \sum_{i=1}^{n} d_{i}=k\right\} \quad(k \in \mathbb{N} \cup\{0\}),
$$

and the partial average $R(n, k)$ for $T_{1}, T_{2}, \ldots, T_{n}$ by

$$
R(n, k)=\frac{1}{|d(n, k)|} \sum_{\vec{d} \in d(n, k)} T_{1}^{d_{1}} T_{2}^{d_{2}} \ldots T_{n}^{d_{n}},
$$

where $|d(n, k)|$ denotes the cardinal number of the set $d(n, k)$ (see [5]). Furthermore, for any $\vec{e}=\left(e_{1}, e_{2}, \cdots, e_{n}\right) \in(\mathbb{N} \cup\{0\})^{n}$, we define the $\vec{e}$-shifted partial average $R(n, k, \vec{e})$ by

$$
R(n, k, \vec{e})=\frac{1}{|d(n, k)|} \sum_{\vec{d} \in d(n, k)} T_{1}^{d_{1}+e_{1}} T_{2}^{d_{2}+e_{2}} \cdots T_{n}^{d_{n}+e_{n}} .
$$

By using this notation, we can get the following result concerning the statement $(* *)$.

Theorem 4. Let $T_{1}, T_{2}, \ldots, T_{n} \in B(\mathcal{H})$ be commuting with each other. Then the following are equivalent:

(1) There exists a positive integer $K$ such that

$$
\sup _{\vec{e}}\|I-R(n, k, \vec{e})\| \leq 1 \quad \text { for all } k \geq K .
$$

(2) There exists a positive integer $K$ such that

$$
\sup _{\vec{e}}\{\|I-R(n, K, \vec{e})\|,\|I-R(n, K+1, \vec{e})\|\} \leq 1 .
$$

(3) There exist a closed subspace $\mathcal{K}$, pairwise commuting nilpotent operators $\left\{N_{i}\right\}$ acting on $\mathcal{K}$ and pairwise commuting positive contractions $\left\{S_{i}\right\}$ acting on $\mathcal{K}^{\perp}$ such that $T_{i}=N_{i}+S_{i}$ for all $i=1,2, \ldots, n$.

Proof. (1) $\Rightarrow(2)$ It is trivial.

$(3) \Rightarrow(1)$ We assume $N_{i}^{n_{i}}=0$. Then for every $k>\sum_{i=1}^{n} n_{i}$ we have

$$
\begin{aligned}
R(n, k, \vec{e}) & =\frac{1}{|d(n, k)|} \sum_{\vec{d} \in d(n, k)} T_{1}^{d_{1}+e_{1}} T_{2}^{d_{2}+e_{2}} \cdots T_{n}^{d_{n}+e_{n}} \\
& =\frac{1}{|d(n, k)|} \sum_{\vec{d} \in d(n, k)} S_{1}^{d_{1}+e_{1}} S_{2}^{d_{2}+e_{2}} \cdots S_{n}^{d_{n}+e_{n}} .
\end{aligned}
$$

Therefore $R(n, k, \vec{e})$ is a positive contraction, which shows $\|I-R(n, k, \vec{e})\| \leq 1$. 
$(2) \Rightarrow(3)$ Let $k$ be equal to $K$ or $K+1$. Since

$$
R(n, k, \vec{e}) R(n, l, \vec{f})=\frac{1}{|d(n, l)|} \sum_{\vec{d} \in d(n, l)} R(n, k, \vec{e}+\vec{f}+\vec{d})
$$

and $\|I-R(n, k, \cdot)\| \leq 1$, we have

$$
\sup _{m \in \mathbb{N}}\left\|I-R(n, k, \vec{e})^{m}\right\| \leq 1
$$

for all $\vec{e} \in(\mathbb{N} \cup\{0\})^{n}$. By Theorem 1 , we have $0 \leq R(n, k, \vec{e}) \leq 1$. We put

$$
\overrightarrow{e_{j}}=(0,0, \cdots, 0, \underbrace{j}_{1}, 0, \cdots, 0) .
$$

The identities

$$
R(n, K+2, \overrightarrow{0})=\frac{1}{|d(n, K+2)|} \sum_{i=1}^{n}|d(n, K+1)| R\left(n, K+1, \overrightarrow{e_{i}}\right)
$$

and

$$
T_{i}^{k+1}=|d(n, k+1)| R(n, k+1, \overrightarrow{0})-\sum_{j \neq i}|d(n, k)| R\left(n, k, \overrightarrow{e_{j}}\right)
$$

imply that $T_{i}^{k+1}$ is self-adjoint for $k=K, K+1$ and $i=1,2, \ldots, n$. By Corollary 3 , $T_{i}$ is represented as the direct sum of a nilpotent operator $N_{i}$ such that $N_{i}^{K+1}=0$ and a self-adjoint operator $S_{i}$. Since $T_{i} T_{j}=T_{j} T_{i}$, we have

$$
S_{i} S_{j}=S_{j} S_{i}, S_{i} N_{j}=N_{j} S_{i}
$$

Let $Q_{i}$ be the orthogonal projection onto $\operatorname{Ker} T_{i}^{k+1}$ and let $P_{i}=I-Q_{i}$. It follows from the property $T_{i} T_{j}=T_{j} T_{i}$ that

$$
Q_{i} Q_{j}=Q_{j} Q_{i}, \operatorname{Ker} S_{i}=Q_{i} \mathcal{H}
$$

If we put $\mathcal{K}=Q_{1} Q_{2} \cdots Q_{n} \mathcal{H}$, then $\mathcal{K}^{\perp}=\left(I-Q_{1}\right) \vee\left(I-Q_{2}\right) \vee \cdots \vee\left(I-Q_{n}\right) \mathcal{H}=$ $\left(I-Q_{1} Q_{2} \cdots Q_{n}\right) \mathcal{H}$.

Let us prove $N_{i}\left(I-Q_{1} Q_{2} \cdots Q_{n}\right)=0$ for all $i$. It is sufficient to prove that $N_{n}\left(I-Q_{1} Q_{2} \cdots Q_{n}\right)=0$. We put

$$
\begin{aligned}
& l=\max \left\{m \mid N_{n}^{m}\left(I-Q_{1} Q_{2} \cdots Q_{n}\right) \neq 0\right\}, \\
& \mathcal{L}=\operatorname{Ker} N_{n}^{l}\left(I-Q_{1} Q_{2} \cdots Q_{n}\right) .
\end{aligned}
$$

We assume $l \geq 1$. Then we can choose a unit vector $x \in \mathcal{L}^{\perp}$ such that $x=$ $\left(I-Q_{1} Q_{2} \cdots Q_{n}\right) x, 0 \neq N_{n}^{l} x \in \operatorname{Ker} T_{n} \subset \mathcal{L}$. Since

$$
\begin{aligned}
& \left\|\left(I-R\left(n, k, \vec{e}+l \overrightarrow{e_{n}}\right)\right) x\right\|^{2} \\
& =\|x\|^{2}-2 R e\left\langle R\left(n, k, \vec{e}+l \overrightarrow{e_{n}}\right) x \mid x\right\rangle+\left\|R\left(n, k, \vec{e}+l \overrightarrow{e_{n}}\right) x\right\|^{2} \\
& =1+\left\|R\left(n, k, \vec{e}+l \overrightarrow{e_{n}}\right) x\right\|^{2}
\end{aligned}
$$


and the assumption $\left\|I-R\left(n, k, \vec{e}+l \overrightarrow{e_{n}}\right)\right\| \leq 1$, we have $R\left(n, k, \vec{e}+l \overrightarrow{e_{n}}\right) x=0, k=$ $K, K+1$. It follows from $x=\left(I-Q_{1} Q_{2} \cdots Q_{n}\right) x$ that, for each $i=1,2, \ldots n-1$,

$$
\begin{aligned}
T_{i}^{K+1} N_{n}^{l} x= & |d(n, K+1)| R(n, K+1, \overrightarrow{0}) N_{n}^{l} x \\
& \quad-\sum_{j \neq i}|d(n, K)| R\left(n, K, \overrightarrow{e_{j}}\right) N_{n}^{l} x \\
=|d(n, K+1)| R\left(n, K+1, l \overrightarrow{e_{n}}\right) x & \quad-\sum_{j \neq i}|d(n, K)| R\left(n, K+1, \overrightarrow{e_{j}}+l \overrightarrow{e_{n}}\right) x \\
= & 0 .
\end{aligned}
$$

Therefore $P_{i} N_{n}^{l} x=0$. Consequently $x \in \operatorname{Ker}_{n}^{l}\left(P_{1} \vee P_{2} \vee \cdots \vee P_{n}\right)=\mathcal{L}$. This contradicts the assumption $l \geq 1$ So we get $N_{n}\left(I-Q_{1} Q_{2} \cdots Q_{n}\right)=0$. Therefore we have $N_{i} \in B\left(Q_{1} \wedge Q_{2} \wedge \cdots \wedge Q_{n} H\right)$ and $S_{i} \in B\left(P_{1} \vee P_{2} \vee \cdots \vee P_{n} H\right)$ for all $i=1,2, \ldots, n$.

It remains to prove $S_{i} \geq 0$. By the Gelfand-Naimark theorem, the $\mathrm{C}^{*}$-algebra generated by $\left\{S_{i}\right\}$ can be identified with the algebra of all the continuous functions on a compact Hausdorff space $\Omega$. In this identification, we can see each $S_{i}$ is a realvalued continuous function on $\Omega$. We put $\mathcal{E}=\left\{\left(f_{1}, f_{2}, \cdots, f_{n}\right) \in(\mathbb{N} \cup\{0\})^{n} \mid f_{i} \geq\right.$ $K, i=1,2, \ldots, n\}$. We can get the positivity of $S_{1}, S_{2}, \cdots, S_{n}$ by the relations

$$
\begin{gathered}
R(n, k, \vec{f})=\frac{1}{|d(n, k)|} \sum_{\vec{d} \in d(n, k)} S_{1}^{d_{1}+f_{1}} S_{2}^{d_{2}+f_{2}} \cdots S_{n}^{d_{n}+f_{n}}, \\
S_{i}^{K+1+f_{i}}=|d(n, K+1)| R(n, K+1, \vec{f})-|d(n, K)| \sum_{j \neq i} R\left(n, K, \vec{f}+\overrightarrow{e_{j}}\right)
\end{gathered}
$$

and

$$
0 \leq R(n, k, \vec{f})
$$

for all $\vec{f} \in \mathcal{E}, k=K, K+1$. This completes the proof.

Remark. If we consider the case $T=T_{1}=T_{2}=\cdots=T_{n}$, then the statement of Theorem 4 implies that of Theorem 1 . Without the assumption that $T_{1}, T_{2}, \cdots, T_{n}$ are pairwise commuting, we cannot show the implication $(2) \Rightarrow(3)$. For example,

$$
T_{1}=\left(\begin{array}{ll}
0 & 1 \\
0 & 0
\end{array}\right), T_{2}=\left(\begin{array}{ll}
1 & 0 \\
0 & 0
\end{array}\right)
$$

are not commuting and the above implication $(2) \Rightarrow(3)$ does not hold for $T_{1}, T_{2}$.

\section{Application of Theorem 1}

Let $A$ be a unital involutive Banach algebra over $\mathbb{C}$. We call $A$ an $A^{*}$-algebra if $A$ has a faithful *-representation on a Hilbert space.

Theorem 5. Let $A$ be a unital $A^{*}$-algebra. If $S$ is a semi-group of $A$ contained in $\{a \in A \mid\|a-I\| \leq 1\}$, then $S$ is abelian. 
Proof. Let $\pi$ be a unital, faithful *-representaion of $A$. Then we have

$$
\begin{aligned}
\|\pi(x)\|^{2} & =\left\|\pi(x)^{*} \pi(x)\right\|=\left\|\pi\left(x^{*} x\right)\right\| \\
& =\sup \left\{\mid \lambda \| \lambda \in S p\left(\pi\left(x^{*} x\right)\right)\right\} \leq \sup \left\{|\lambda| \mid \lambda \in S p\left(x^{*} x\right)\right\} \\
& =\lim _{n \rightarrow \infty}\left\|\left(x^{*} x\right)^{n}\right\|^{1 / n} \leq\left\|x^{*} x\right\| \leq\|x\|^{2} .
\end{aligned}
$$

Therefore the semi-group $\pi(S)$ is contained in $\{x \in \pi(A) \mid\|x-I\| \leq 1\}$. For $a \in S$, $\left\|\pi(a)^{n}-I\right\| \leq 1$ for all $n \in \mathbb{N} \cup\{0\}$. Using Theorem 1 , we have $\pi(a), \pi(b)$ and $\pi(a b)$ are positive contractions for $a, b \in S$. Since $\pi$ is faithful and the calculation,

$$
\pi(a) \pi(b)=\pi(a b)=\pi(a b)^{*}=\pi(b)^{*} \pi(a)^{*}=\pi(b) \pi(a),
$$

we have that $S$ is abelian.

If $A$ is a unital involutive Banach algebra and not an $A^{*}$-algebra, then the above theorem is not necessarily valid. In fact, we have the following example:

Example. For an element $(\alpha, \beta)$ of $\mathbb{C}^{2}$, we consider the following norms:

$$
\|(\alpha, \beta)\|_{1}=|\alpha|+|\beta|, \quad\|(\alpha, \beta)\|_{\infty}=\max \{|\alpha|,|\beta|\} .
$$

Let $x$ be an element of $M_{2}(\mathbb{C})$. We can regard $x$ as a bounded linear map from $\left(\mathbb{C}^{2},\|\cdot\|_{1}\right)\left(\operatorname{resp} .\left(\mathbb{C}^{2},\|\cdot\|_{\infty}\right)\right)$ to $\left(\mathbb{C}^{2},\|\cdot\|_{1}\right)\left(\operatorname{resp} .\left(\mathbb{C}^{2},\|\cdot\|_{\infty}\right)\right)$, and consider the following norms:

$$
\begin{array}{cl}
\|x\|_{1}=\max \{|a|+|c|,|b|+|d|\} & \left(x \in B\left(\mathbb{C}^{2},\|\cdot\|_{1}\right)\right), \\
\|x\|_{\infty}=\max \{|a|+|b|,|c|+|d|\} \quad\left(x \in B\left(\mathbb{C}^{2},\|\cdot\|_{\infty}\right)\right),
\end{array}
$$

where $x$ has the form $\left(\begin{array}{ll}a & b \\ c & d\end{array}\right)$. We define the conjugate linear map $s$ from $M_{2}(\mathbb{C})$ to $M_{2}(\mathbb{C})$ by

$$
s(x)=s\left(\begin{array}{ll}
a & b \\
c & d
\end{array}\right)=\left(\begin{array}{ll}
\bar{a} & \bar{c} \\
\bar{b} & \bar{d}
\end{array}\right) .
$$

Then we can easily check $\|s(x)\|_{\infty}=\|x\|_{1}$ and $\|s(x)\|_{1}=\|x\|_{\infty}$. We consider the algebra $A=M_{2}(\mathbb{C}) \oplus M_{2}(\mathbb{C})$ and define the norm and the involution on $A$ by

$$
\|(x, y)\|=\max \left\{\|x\|_{1},\|y\|_{\infty}\right\} \text { and }(x, y)^{*}=(s(y), s(x)),
$$

where $x, y \in M_{2}(\mathbb{C})$. Then $A$ becomes an involutive Banach algebra. We set $S$ to be the semi-group of $A$ generated by $\left(x_{1}, 0\right)$ and $\left(x_{2}, 0\right)$, where $x_{1}=\left(\begin{array}{ll}0 & 1 \\ 0 & 1\end{array}\right)$ and $x_{2}=\left(\begin{array}{ll}0 & 0 \\ 0 & 1\end{array}\right)$. In fact, $S$ consists of only two elements $\left(x_{1}, 0\right),\left(x_{2}, 0\right)$. Clearly $S$ is not abelian and

$$
\left\|(I, I)-\left(x_{1}, 0\right)\right\|,\left\|(I, I)-\left(x_{2}, 0\right)\right\| \leq 1
$$




\section{REFERENCES}

1. Cox, R. H., Matrices all whose powers lie close to the identity, Amer. Math. Monthly $\mathbf{7 3}$ (1966), 813.

2. DePrima, C. R., Richard, B. K., A Characterrization of the Positive Cone of $B(\mathcal{H})$, Indiana Univ. Math. Jour. 23 (1973), 1085-1087. MR 47:4052

3. Halmos, P. R., A Hilbert Space Problem Book Second Ed., Springer-Verlag, New York, 1982. MR 36:5743

4. Kato T., Some mapping theorems for the numerical range, Proc. Japan Acad. 41 (1965), 652-655.

5. Nagisa M., Wada S., An extension of the mean ergodic theorem, to appear in Math. Japon. (Vol. 48, No. 1).

6. Nakamura M., Yoshida M., On a generalization of a theorem of Cox, Proc. Japan Acad. 43 (1967), 108-110. MR 36:708

7. Takesaki, M., Theory of Operator Algebras I, Springer-Verlag, 1979. MR 81e:46038

Department of Mathematics and Informatics, Faculty of Science, Chiba University 1-33 YAYOI-CHO, InAGe-Ku Chiba, 263, JaPAN

E-mail address: nagisa@math.s.chiba-u.ac.jp

Department of Information and Computer Engineering, Kisarazu National College of Technology 2-11-1 Kiyomidai-Higashi, Kisarazu, Chiba, 292, Japan

E-mail address: wada@gokumi.j.kisarazu.ac.jp 